\title{
Design practice of interactive imaging art in the new media art-Taking "Ink-wash Tai Chi" as an example
}

\author{
Lu Geng ${ }^{1, *}$, and Lei $\mathrm{CaO}^{2}$ \\ ${ }^{1}$ College of Sino-Korea Multimedia Design, Shanghai University of Engineering Sciences, Shanghai, \\ China \\ ${ }^{2}$ College of Electronic and Electrical Engineering,Shanghai University of Engineering Sciences, \\ Shanghai, China
}

\begin{abstract}
In order to be accepted by the extensive public, the traditional culture requires digital media interactive methods featuring "diversified sensual experiences" and "interactivity" to adapt better to the development tendency of cultural communication under the background of new media. The design practice, with Tai Chi culture as the content and using motion data captured by infrared sensor Kinect combined with digital art creation platform Processing as the technical measures to implement interactive imaging. The work will give interactive experience to the experiencer and promote the spread of Tai Chi culture.
\end{abstract}

\section{Interactive imaging art overview}

The interactive imaging art, as a new art form emerged recently in the world, belongs to the subject of new media art. The new media refer to the media based on digital technology and featuring interactive communication and creative forms[1]. The interactive imaging art is the best manifestation of the interactivity of new media art. It grew out of but is different from the video art and computer graphic art[2].This new art form requires the strong support of information technology of the time. The interactive imaging art depends on various sensors to capture the spatial or moving data, which will then be collected and put into computer for calculating and processing. In this way, uncertain imaging feedbacks with random beauty are gained. This interactive form breaks the previous rigid and non-interactive art form. The participation of people and environment can change many characteristics and even the meanings of a work. McLuhan said in the book of understanding media that "Media is an extension of people," and people is the world's most primitive information media[3]. The interactive imaging can encourage the emotional exchanges between people and narrow the distance between people and the art.

In the Video Place, an interactive imaging device work by Myron Krueger in the 70s, the first system allowing users to interact with virtual objects was created. The device mixed the imaging pictures of the users with other images through a computer. This is significant as the initial appearance of interactive imaging. This work created a new art

*Corresponding author: genglu_2005@163.com 
form based on the "creator - computer system - experiencer" interaction. Taking the images of the audiences as an important part of the work, this interaction mode will become the basic model for many of the interactive media works in the future[4].

\section{Interactive form in interactive imaging art}

As an interactive method, interactive image art mainly uses interactive hardware and programming languages a human-machine interface to perform "dialogue" with the experiencer. There are three commonly used methods in interactive images: touch interaction, gesture and action interaction, and voice interaction. Gesture and action interaction are easy-to-understand languages that are consistent with popular cognitive habits, enabling natural interactions between humans and computers.

With the device interactive work named Quantum Space, the movements of experiencers who entered the space are identified by the Xtion depth camera and computer system to project one or more digital figure images on the digital wall in time. These digital figures are momentary, now colorful swirls, now geometrical neon, and now particle system, parting and gathering in different shapes. The device generates shapes and colors based on particle physical modelling and then casts digital figures in various postures(see figure 1). As the designer Sodazot explained: "In the quantum space, you are divided into light quantum and empowered with the force to communicate with the universe".

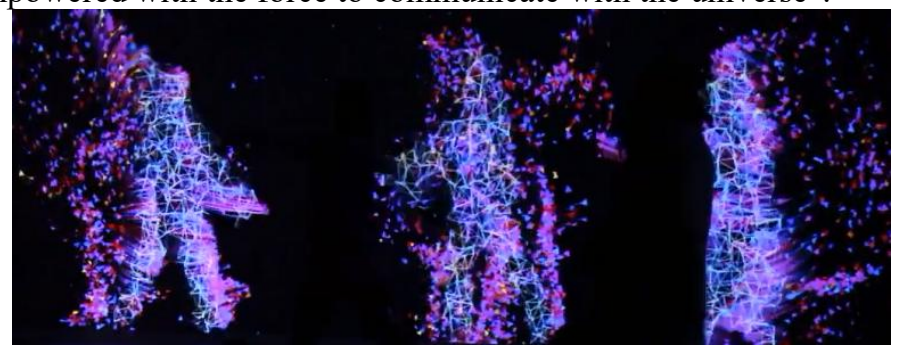

Fig. 1. Imaging of a Quantum Space Device Interactive Work.

Established in 2001, TeamLab, a technology art institution from Japan, created many immersion works featuring mixed lights and shadows and fabulous beauty. For example, the theme work" Flower Forest, Lost and Immersed" builds a fancy world for the audience. The exhibition hall is all dark when empty. If someone steps into the hall, flowers will bloom on his or her body and spread to the feet, and then link with the flowers on the next person; the flowers bloom and wither automatically, in cycles without an end; when the visitor is sitting or standing still, flowers are in extraordinary bloom; when visitors are leaving, flowers begin to wither and disappear. Then the hall returns to darkness(see figure 2). The TeamLab team created this work to break the boundaries in modern people's minds, those between themselves and the world and between the world and others, enlightening the future living space of digital art.

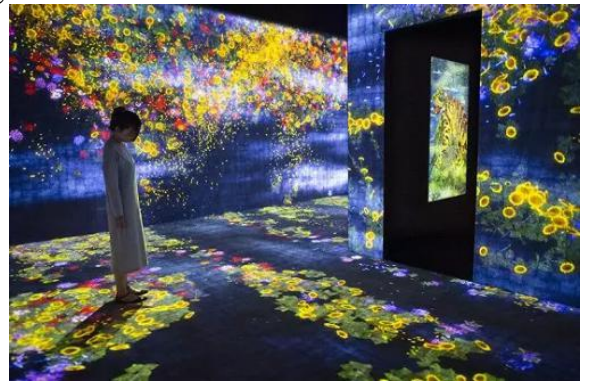

Fig. 2. An interactive Imaging Work from TeamLab,the "Flower Forest, Lost and Immersed". 


\section{Design practice of the interactive imaging work named "Ink Painting Tai Chi"}

\subsection{Work contents}

The Tai Chi culture, as a representative of Chinese traditional culture, is very famous at home and abroad. The Tai Chi exercise is also the most popular Chinese traditional fitness project in the world. Chinese Tai Chi, applying to be one of the world intangible cultural heritages, requires urgent improvement in exposure and influence on the world cultural stage.

Driven by the social background of cross-border integration and multicomponent integration, the traditional arts are all trying to become diversified, in order to keep a foothold and make improvement. There are practices of interaction + dance, interaction + drama, and interaction+ music, integrating technology into the traditional arts and realizing stronger participation and interactivity. The Ink Painting Tai Chi is a design practice work trying to combine interaction with Tai Chi. Through the picture effects like ink painting, it combines the movements of Tai Chi exercise with twenty-four forms with the Tai Chi theories and shows them by means of interactive imaging art, reflecting the ideas of Tai Chi in four interactive aspects, namely the opening, developing, changing and concluding.

Opening: the start of the Tai Chi exercise with twenty-four forms requires concentration, as calm as the mountains. The work simulates the ink painting mountains with Processing. The opening movements in Tai Chi, together with breathes, will control the ups and downs of the mountains. Breathing in for ups and breathing out for downs. There is a sun rising behind the mountains.

Developing: smooth connection, natural linkage, and movements in line with will. The filmy figure of the experiencer shows up in the work. When the experiencer is moving from left to right slowly, the postures can be seen in the screen. The moving traces of hands stay for a while like the smoky particles. The "Qi" of Tai Chi is represented by particles, displaying the relaxed and full movements in Tai Chi.

Changing: variegated, motion-stillness integrated, and virtual-real transformable. There are two interactions in this part. Firstly, when the experiencer is moving, each movement is recorded and, along with the time, the records are made into the static shadows of a series of different movements. When the experiencer is still, the shadows begin to move. Secondly, the pictures show the outlines of the twenty-four forms in addition to the shadow of the experiencer. If the experiencer's movements match with the form outlines, it will turn to the next form.

Conclusion: the ending should be with integrated harmony, and you will know about Yin and Yang if you can not only open but also conclude. The figure of the experiencer is no longer concrete. It can interact with the numerous particles in the pictures, which do not disappear or rest and will gather into a shape of Tai Chi in the end.

\subsection{Technical structure}

The Tai Chi movements and body images of the experiencer are sensed in time by the Kinect depth camera. The data collected will be sent to a computer for the processing by SimpleOpenNI, an open human-computer language interactive framework. The computer can identify the body location and the joint positions in the 3D space through the original depth data. According to the feedbacks of different movement signal sources, image changes preset by the Processing are casted synchronously. The real-time interactions between the experiencer's body and the images are realized(see figure 3 ). 


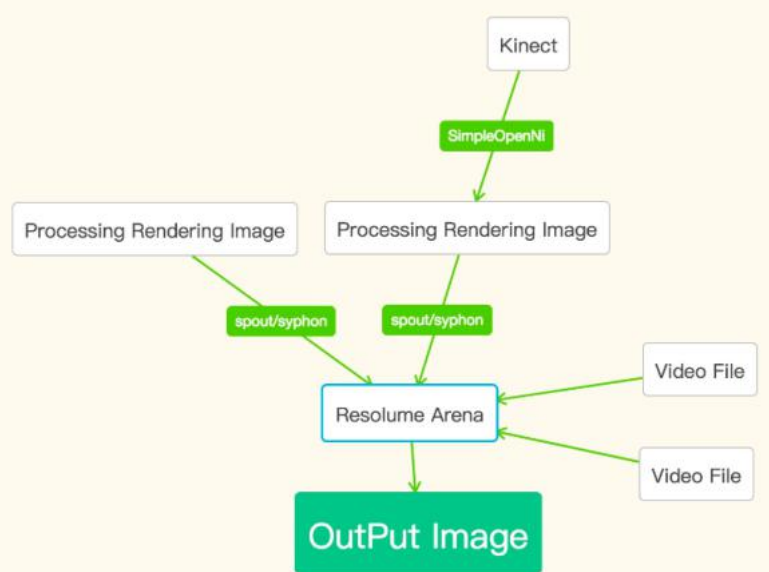

Fig. 3. Technical Structure of the Work.

The User mechanism of the SimpleOpenNI enables the interactions between more local users. Once a new user is identified, the response function onNewUser(), is motivated to register the user ID and start the Tracking Skeleton. The user outlines traced by the device is stored in the form of binary image matrix, which can be used through userMap $($ ) function in the form of arrays.

\subsection{Key techniques implementation approaches and results show}

\subsubsection{Ink painting mountains simulation by processing}

Realize the overlaps and fluctuations of mountains with a self-defined class, which consist of: image variables to record a transverse mountain, coordinate of the mountains in the picture, two-dimensional variables to generate the Perlin noise of the mountains, and the dynamic moving distances of mountains. A transverse mountain is completed through the drawing function and the Perlin noise in Processing. The program records and shows the mountain pictures generated along with the ups and downs of the experiencer's hands, creating mountain images of different heights and distances[5] (see figure 4).
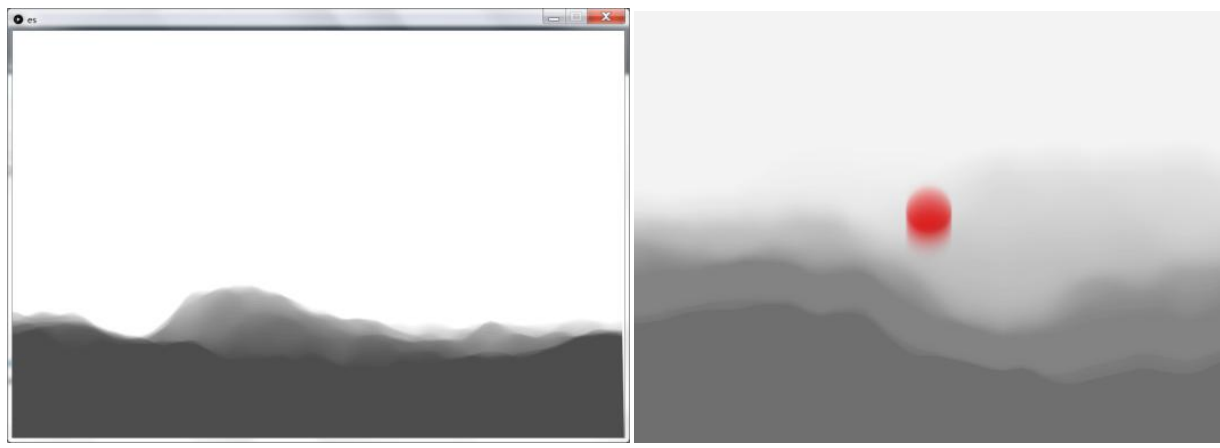

Fig. 4. Ink Painting Mountains.

\subsubsection{Haze effects simulation by processing}

Haze effects are realized by identifying two classes. One for haze particle effects realization, 
setting the initial moving speed and directions of particles with the random numbers produced by random Gaussian(). The other for particle swarm management, controlling the lifespan of particles with a member variable of Lifespan, regulating the running of each particle and removing those ended particles(see figure 5).

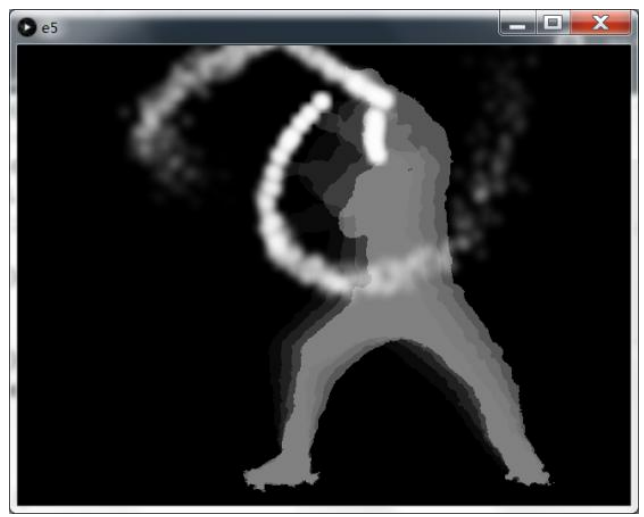

Fig. 5. Haze Particles.

\subsubsection{Realization of multi-linear record and display through processing}

The recording process is divided into four stages: In the reset stage, all relevant parameters are reset and the second stage will come in the next frame. In the second stage of recording, the experiencer's body moving process is recorded and the frame will be displayed if it is the integral multiple of the space, so that the experiencer images will stay for a long time. The third is the pause stage. Since estimation of the duration of experiencer's each movement is key to the implementation of recording, a threshold value is set in order to stop the recording when the experiencer is holding a posture. The recording will stop when the holding time surpasses the threshold value and continue when the user moves again. If the pause time reaches the threshold value, the display will begin. The last is the multi-linear display stage. The breaking points are displayed at the same time and it will return to the first stage until the first breaking point has been displayed(see figure 6).

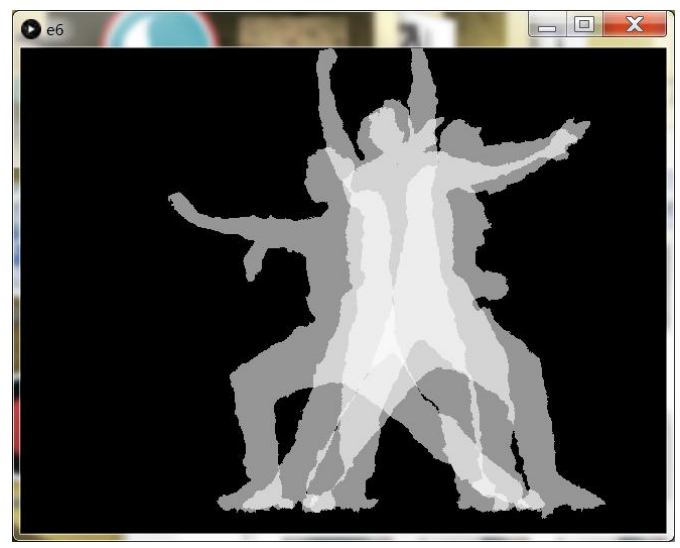

Fig. 6. Multi-linear Recording.

\subsubsection{Particle Tai Chi images realized through processing}

In order to realize the production of Tai Chi images with particles, a picture of Tai Chi in 
the format of png or jpg should be prepared in advance. During the reviewing of all pixels of the picture, the pixel is identified as the coordinate of a particle if its lightness is lower than the threshold value. And the identified coordinates are used for the recovery of Tai Chi images. The number of particles depends on their intervals. Before the Tai Chi images are produced, interactions between the particles and figures are achieved by using repulsive particles(see figure7 and 8).

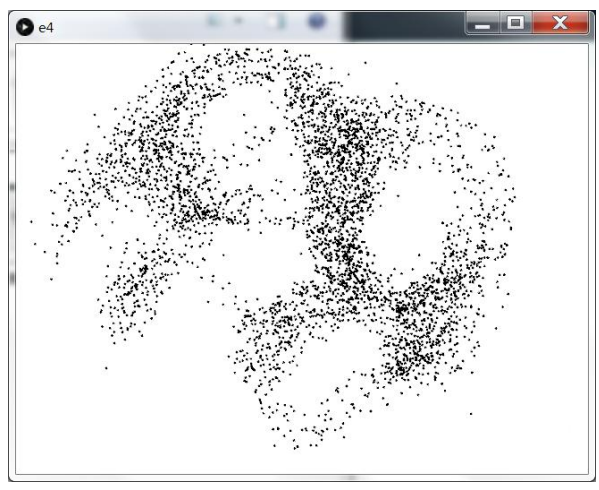

Fig. 7. Interactions between the Particles and Figures.

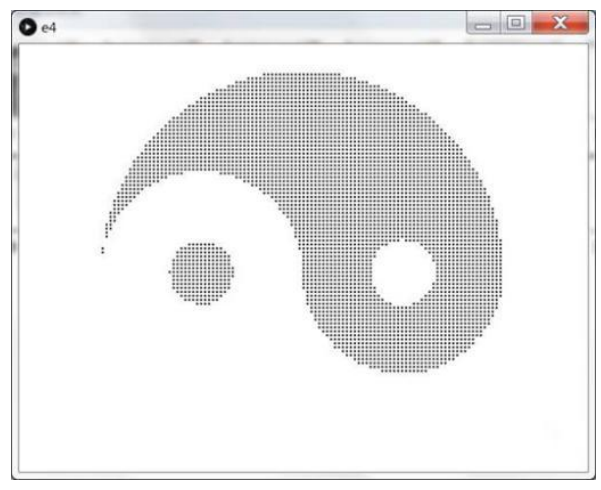

Fig. 8. Particles Aggregate into Tai Chi Images.

\section{Conclusion}

Interactive imaging is one of the expressions of new media arts. It has strong connectivity and interaction. Through the different connections, integration, interaction and transformation between the experiencer and the work, the image is finally presented. The imaging at this time is a work created by the creator and the experiencer. It is not only the image itself with the meaning of artistic creation, but also an extension of people's actual experience. Through the design practice of this article, it is also proved that in the context of cultural communication design in the digital context, it is necessary to integrate technology and art reasonably, establish emotions through interaction and exchange, enhance the sense of cultural identity, and create a dialogue-style cultural experience.

\section{References}

1. Baidu Encyclopedia 2017 New Media http://baike.baidu.com/view/339017.htm

2. Hua Yang 2009 New Media Art Interactive Imaging Installation Art (China: Shandong Fine Arts Publishing House)

3. Marshall McLuhan 2011 Understanding Media: The Extensions of Man (Canada: Yilin Press)

4. Ran Zhang 2013 Real-time Interactive Image Rendering Based on Processing Platform (China: Beijing University of Technology)

5. Daniel Shiffman 2017 Learning Processing (China: China Machine Press) 